\title{
Test and Analysis of High Voltage and High Frequency Capacitors Concerning Frequency Characteristics
}

\author{
Heisik Kim ${ }^{1,}$, Seoyeon Won ${ }^{1}$, Daeseong Hwang ${ }^{2}$ and Kyeongjin Lee ${ }^{2}$ \\ ${ }^{1}$ Electrical and Computer Engineering Department, University of Seoul, Korea \\ ${ }^{2}$ Technology Research Institute, Daedong Condensor Co. Korea \\ ${ }^{*}$ Corresponding Author
}

\begin{abstract}
The high frequency and high voltage capacitor was designed and tested and produced with precision technology. The film winding machine was installed and installed to produce new designed capacitors. By winding capacitor core, MPP (Metalized Film on Propylene Film) and PP (Propylene Film) or Aluminum film were feed together in multi-layers into the roll with very adjusted and controlled tension force. By testing to analysis of new designed various capacitors with different tension methods. The resonance frequency and allowable frequency range and durable high voltage of all new designed capacitor core roll were tested. The test result with prototype roll showed allowable frequency of $700 \mathrm{KHz}$ and 450 Volt AC. They were approved enough proper performance by the detailed test procedure to be applied for high power induction heater LC circuit of industrial heating facilities.
\end{abstract}

Keywords-capacitor; test; frequency characteristics; high power; LC circuit; induction heater; film condenser; water cooled capacitor; rolling MPP

\section{INTRODUCTION}

The conduction cooled capacitor for LC oscillation circuit of induction heating facilities is very important electrical component. Induction heating and dryer are applied more in industrial plants for its clean operation and rapid heat treatment comparing to the conventional heating equipment based on the burning process of the carbon or carbon hydrate oxidation. Electrical induction heating equipment is operated in high frequency range of $200 \mathrm{KHz}$ to $700 \mathrm{KHz}$ with energy of 150 KVAR to 400 KVR per unit capacitor. Main problem in these capacitors for induction heating circuit is to cool down continuously the much heat generation from the capacitor core side. The Propylene film of dielectric material layers is a kind of polymer materials that has weakness point against temperature increasement. If the temperature goes higher than some limitation of $120 \mathrm{C}$ grade, its chemical structure inside would be break down and loose the necessary dielectricity value and also electrical insulation resistivity between both metal films. So it must be thrown away to change new capacitor. One unit capacitor of conduction cooled high power capacitor costs about $\$ 100$ per unit. So the capacitor damage by high temperature must be protected with high caution with cooling operation with temperature measurement continuously during its operation.

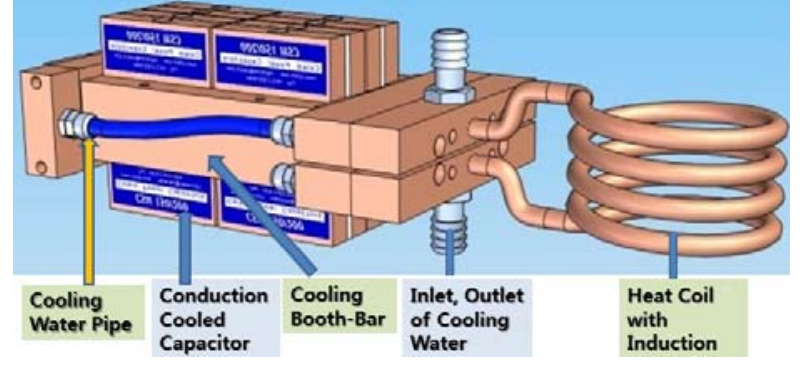

FIGURE I.

INDUSTRIAL INDUCTION HEATING EQUIPMENT WITH WATER COOLED HIGH POWER CAPACITOR

The Figure 1 shows the schematic diagram of induction heating equipment with water cooled high power capacitor application. The cold water passing through the hole in the capacitor pole rod of copper material allows cooling down the high temperature heat generated by alternative electrical current.

\section{Testing Process AND EQUIPMENT}

For testing of spectral characteristics of developed capacitors model, the HIOKI LCR impedance tester was applied. The Figure 2 shows the photo of experimental equipment to measure the capacitance change on sweep frequency.

Before starting measurement of capacitance, loss coefficient, ESR and other interesting parameters, it must be calibrated by short calibration and open calibration procedure, both. For high precision measurement, the effect of probe cable capacitance and resistance value must be known to the LCR meter by the prior calibration process. Hioki LCR tester requested probe cable of 75 Ohms.

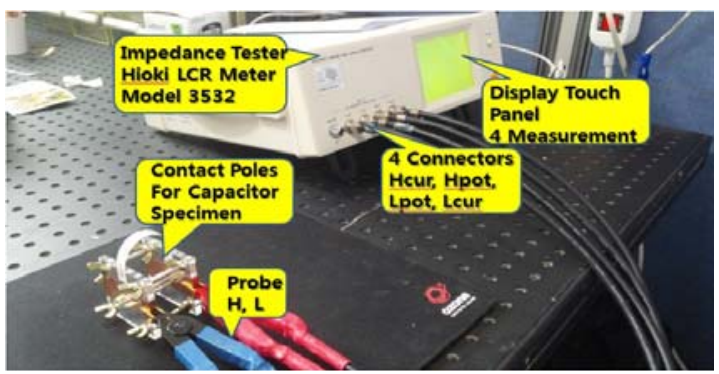

FIGURE II. HIOKI LCR METER TO TEST CAPACITANCE MEASUREMENT OF VARIOUS WINDING CAPACITOR TYPES. 


\section{Result OF Testing VARIOUS CAPACITOR Roll SPECIMENS}

For the test of capacitor characteristics of durable voltage and durable frequency, various roll type and various film layers design construction forms were made by this experiment phase.

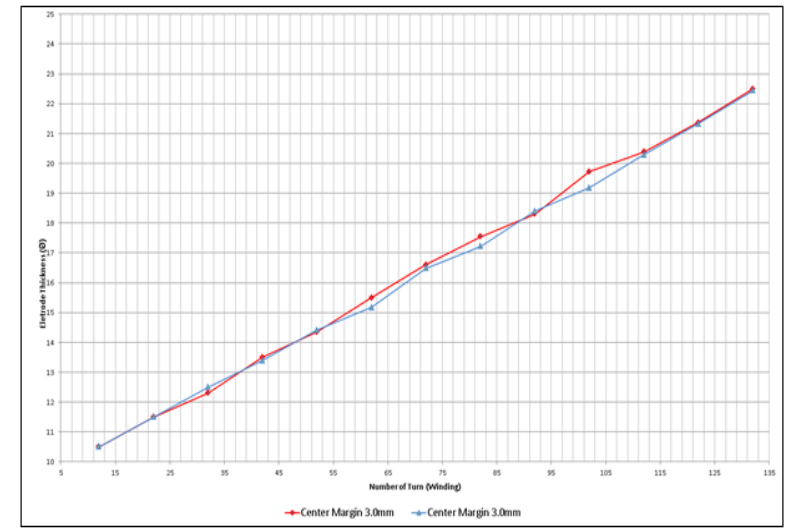

FIGURE III. LESS CHANGE OF CAPACITANCE VALUES ON TWO DIFFERENT CENTER MARGIN OF 3 MM AN D 2.5 MM

In Figure 3, Two different center margin film types of $3.0 \mathrm{~mm}$ and $2.5 \mathrm{~mm}$ design capacitor showed no big different capacitance values. The capacitance values are increasing proportional to the roll numbers of film condenser type.

The capacitor film roll turn numbers of the film electrode were 12 roll number as start initial type and then increased 10 roll times by every specimen until to 132 times roll number. So, 13 different type roll capacitors were rolled by using capacitor winding machine. They were tested as test experimental specimens. In Figure 4, the measured capacitance values were proportional to the roll turn numbers.

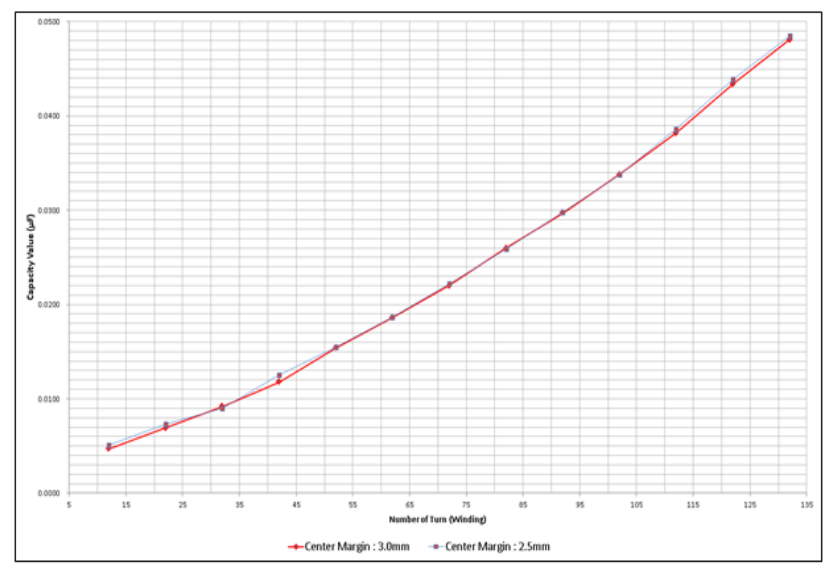

FIGURE IV.

INCREASED CAPACITANCE VALUES

PROPORTIONAL TO THE ROLL FILM TURN NUMBERS OF CAPACITORS

The slight curve but not straight proportional line of capacitance values comes due to the increased radius or diameters by increasing the total capacitor film length.

\section{CONCLUSION}

The developed capacitors rolled with various roll lengths and layers construction design and various tension conditions were tested to get the best performance. The capacitance value in same type increased proportional to the roll turn numbers. With the test of change of capacitance according to the number of turn, the tested capacitance value is proportional to the turn number. Higher rotation speed of rolling showed less durable voltage limit. Stronger tension gave higher capacitance value with smaller distance between conductive Aluminum film with compressed dielectric material Propylene film thickness. Rolling process parameters affect much onto the capacitor's characteristics. The rolled sample capacitors allowed $750 \mathrm{KHz}$ frequency and 450 Volt limit. It showed the enough application with 150 KVAR capacitor for induction heating facilities in industry.

\section{ACKNOWLEDGMENT}

The research was supported by Small and Medium Business Administration. "SMBA Technology Development Project (No. S2164405)" Title "Development of Highfrequency Conduction-Cooled High Capacity (150kVar) Capacitors for Using Heat Treatment Industries".

\section{REFERENCES}

[1] Ralph M. Kerrigan and Bob Kropiewnicki, "Film Capacitor Thermal Strategies Increase Power Density”, CARTS-RMK, COMPONENTS TECHNOLOGY INSTITUTE INC., pp. 163-168, Vol.28 No, 2008

[2] Mr. Boonchoo Somboonepen, "Design and Test of Vegetable Oil Impregnated Polypropylene Film Capacitors, Master’s Thesis, 2007

[3] J.D. Park, H.R. Kwak, H.Y. Park, and J.W. Jung, "Dielectric Characteristics of Composite Dielectric Film for Pulsed Power Capacitors”, KIEE, KIEE Summer Conference, pp. 1661-1663, July 2001

[4] J.R. Yoon, Y.K. Kim, S.W. Lee, and H.Y. Lee, "The Design and Reliability Evaluation of Metallized Film Capacitor for Power Electronic Applications”, J.KIEEME, KIEEME, Vol.24, No. 5, pp. 381-386, May 2011

[5] J.R. Yoon, Y.K. Kim, "Design and Fabrication of Metalized Film Capacitor for Power Electronics” KIEE, KIEE Summer Conference, pp. 14-17, July 2009

[6] Yasunobu Yoshida, Takaashi Muraoka, "The Development of High Voltage Power Capacitor Technology in Japan", IEEE Electrical Insulation Magazine, Vol.11, No.1, pp. 33-45, 1995 